22

\title{
Изображающий спектрограф нормального падения на основе апериодической сферической решетки для вакуумной области спектра
}

\author{
(C) Е.А. Вишняков ${ }^{1}$, А.О. Колесников ${ }^{1,2}$, Е.Н. Рагозин ${ }^{1,2}$, А.Н. Шатохин ${ }^{1,2}$ \\ ${ }^{1}$ Физический институт им. П.Н. Лебедева РАН, \\ 119991 Москва, Россия \\ ${ }^{2}$ Московский фризико-технический институт (государственный университет), \\ 141707 Долгопрудный, Россия \\ ฯ e-mail: alexey6180@gmail.com, enragozin@gmail.com
}

Поступила в редакцию 18.01.2018 г.

В окончательной редакции 24.02.2018 г.

\begin{abstract}
Рассмотрена возможность компенсации астигматизма в широком интервале длин волн в схемах нормального падения на основе сферической дифракционной решетки с переменным шагом. Рассчитаны две схемы изображающих спектрографов с габаритами около 1 и $5 \mathrm{~m}$ для диапазонов длин волн $820-1690 \AA$ и 980-1520 , работающих в первом внешнем и первом внутреннем порядках дифракции соответственно. Показано, что преимуществом внешнего порядка дифракции является широкий (более октавы) спектральный диапазон при относительно компактных размерах прибора. Схемы, работающие во внутренних порядках дифракции, обладают лучшими изображающими характеристиками, но для поддержания ширины диапазона длин волн на уровне октавы требуются большие габариты прибора. Проведено сравнение вышеперечисленных схем со схемами на основе решеток с криволинейными штрихами. Показано, что решетки с переменным шагом дают выигрыш в предельном пространственном разрешении, в то время как решетки с криволинейными штрихами позволяют достичь лучшего предельного спектрального разрешения. Схемы рассмотренного типа могут быть применены в мягком рентгеновском диапазоне при использовании дифракционных решеток с многослойным покрытием.
\end{abstract}

DOI: $10.21883 /$ OS.2018.11.46845.14-18

\section{Введение}

Построение спектральных изображений высокого разрешения в вакуумной ультрафиолетовой (ВУФ) и мягкой рентгеновской областях спектра представляет собой важную, но трудную задачу. В этом диапазоне лежат спектры атомов и ионов, возбуждаемых в электрических разрядах, лазерной плазме, солнечной атмосфере, а также высокие гармоники лазерного излучения. В ряде экспериментов требуется пространственное разрешение $\sim 10 \mu \mathrm{m}$ и даже $\sim 1 \mu \mathrm{m}$. Примерами могут служить, например, компактные источники излучения, получаемые в экспериментах по генерации высоких гармоник в релятивистской лазерной плазме под действием излучения мультитераваттного фемтосекундного лазера [1] и генерация коротковолнового излучения при отражении излучения Ті:сапфирового лазера от релятивистской плазменной волны, возбуждаемой в импульсной струе гелия (релятивистское „летящее зеркало“) [2].

Поскольку ВУФ излучение сильно поглощается в воздухе и большинстве материалов, для построения спектральных изображений в этом диапазоне используются отражательная и дифракционная фокусирующая оптика $[3,4]$. Наиболее часто в качестве одновременно фокусирующего и диспергирующего элемента в схемах дифракционных ВУФ спектрометров служит вогнутая дифракционная решетка. Штрихи классической сферической дифракционной решетки образуются сечениями сферы параллельными эквидистантными плоскостями. Если расположить входную щель на окружности, проходящей через центр апертуры решетки и через центр ее кривизны, то ее спектральные изображения также будут находиться на этой окружности (которая получила название круга Роуланда). Эта схема, предложенная Роуландом в 1882 г. [5], до сих пор часто используется в спектроскопии ВУФ диапазона. Если через центр кривизны классической решетки провести касательную к кругу Роуланда, то любая точка этой касательной будет фокусироваться в вертикальном направлении и изображаться в виде горизонтального астигматического отрезка на этой же касательной. Вблизи точки касания круг Роуланда и вертикальный фокус практически совпадают, что дало возможность Сирксу (при расположении входной щели на круге Роуланда, а источника - на касательной) получить стигматический спектр в узком спектральном диапазоне [6].

Между тем, характерное расстояние между горизонтальным (спектральным) и вертикальным фокусами в роуландовской схеме нормального падения с классической решеткой метрового радиуса и частотой штрихов $600 \mathrm{~mm}^{-1}$ составляет $10-20 \mathrm{~mm}$ на длине волны $\sim 1000 \AA$. При высоте решетки $5 \mathrm{~cm}$ это дает астигматическое размытие $0.5-1 \mathrm{~mm}$.

В 1893 г. М. Корню указал, что монотонное изменение шага решетки по ее апертуре приводит к изменению кривизны волнового фронта дифрагированных пучков и 
положения спектрального фокуса [7]. Он обнаружил, что плоская решетка с требуемым систематическим изменением шага, используемая в коллимированном падающем пучке, будет фокусировать дифрагированный пучок. В настоящее время решетки с шагом, изменяющимся по заданному закону, получили название varied line-space gratings (VLS-решетки). Однако почти в течение века усилия изготовителей решеток были направлены на поддержание максимальной эквидистантности штрихов, так как эквидистантность штрихов классических решеток означала высокую спектральную разрешающую способность и ассоциировалась с их качеством.

В 1970 г. Ф.М. Герасимов сообщил об изготовлении дифракционной решетки с переменным шагом и ее применении для компенсации астигматизма в схеме нормального падения [8]. Переменный шаг штрихов позволил изменить положение спектрального фокуса и добиться пересечения модифицированной горизонтальной фокальной кривой (которая теперь не совпадает с кругом Роуланда) с осью симметрии решетки (вертикальной фокальной кривой) в двух точках. В одной из таких точек размещалась входная щель, в окрестности другой наблюдался спектр. Такая схема прибора позволяла получить практический стигматизм в окрестности одной длины волны.

К 1980 г. Т. Харада разработал механический гравировальный станок, позволяющий нарезать плоские и вогнутые решетки с частотой штрихов $300-3000 \mathrm{~mm}^{-1}$ и минимальным инкрементом шага $0.2 \AA$ [9]. Тогда же он впервые рассчитал спектрограф скользящего падения с плоским полем на коротковолновый диапазон длин волн 50-200 А. В 1983 г. он продемонстрировал прибор в действии при регистрации спектров лазерной плазмы [10]. В приборе использовалась гравированная решетка, шаг которой менялся от 0.99 до $0.69 \mu \mathrm{m}$ на апертуре $50 \mathrm{~mm}$. Этот прибор стал коммерчески доступен и известен как спектрограф Харады. После усовершенствования гравировального станка Харада смог нарезать решетки с частотой штрихов до $\sim 10^{4} \mathrm{~mm}^{-1}$.

Позже Т. Харада и др. рассчитали стигматический спектрограф нормального падения высокого разрешения на диапазон длин волн $250-290 \AA$ для японского спутника Solar-B/Hinode [11]. В работе рассматривались две оптические схемы: на основе сферической VLS-решетки и на основе периодической тороидальной решетки. Первая схема являлась дальнейшим развитием идеи Герасимова, но не предполагала расположения входной щели в одной из точек пересечения модифицированной спектральной фокальной кривой с осью симметрии решетки. Это позволяло получить стигматизм уже на двух длинах волн и компенсировать его в спектральном интервале, включающем в себя обе эти длины волны. Во второй схеме две точки пересечения спектрального и вертикального фокусов обеспечивались тороидальной формой решетки. Первая схема продемонстрировала лучшие изображающие характеристики.
Другой возможностью компенсации астигматизма в схемах нормального падения является использование периодической решетки с криволинейными штрихами. Штрихи такой решетки являются пересечениями сферической поверхности решетки с одинаковыми вертикальными круговыми цилиндрами, оси которых лежат в главной плоскости решетки и располагаются параллельно центральной нормали решетки на одинаковых расстояниях друг от друга [12]. Радиус кривизны штриха $R_{g}$ - это радиус окружности, на которой построены эти цилиндры. Такие решетки отображают спектр на круг Роуланда и могут применяться в классических схемах вместо обычных решеток. Но криволинейные штрихи приводят к изменению формы кривой пространственного фокуса, что дает возможность скомпенсировать астигматизм при выбранном положении входной щели и выбранной длине волны. Частный случай таких решеток, дающих одну из стигматических точек в центре кривизны решетки и компенсирующих астигматизм в окрестности одной длины волны, упоминается Герасимовым [13] в 1979 г. Более общий случай предполагает компенсацию астигматизма в пределах всего рабочего спектрального диапазона и был упомянут в работе [14].

В настоящей работе ставилась цель рассмотреть возможность компенсации астигматизма в схемах нормального падения на основе сферической VLS-решетки в широком спектральном интервале, проанализировать изображающие характеристики этих схем и рассчитать стигматические спектрографы нормального падения, охватывающие область длин волн шириной около октавы с центром вблизи $1250 \AA$. В дополнение также была рассмотрена возможность компенсации астигматизма в схемах на основе вогнутой решетки с эквидистантными криволинейными штрихами.

\section{Фокальные кривые сферической VLS-решетки}

Рассмотрим дифракцию в главной плоскости сферической VLS-решетки (рис. 1,a). Поместим начало координат (точку $O$ ) в центр ее апертуры. Направим ось $O x$ вдоль центральной нормали решетки в сторону центра кривизны ее поверхности (точку $C$ ), а ось $O y$ вдоль касательной перпендикулярно штрихам. Пусть пространственная частота штрихов VLS-решетки линейно зависит от координаты $y$ :

$$
p(y)=p_{0}+p_{1} y,
$$

причем $d n / d y=p(y)$, где $n-$ номер штриха. Расположим в главной плоскости точечный источник (точку $A$ ) и его спектральное изображение (точку $B$ ). Обозначим длину отрезка $A O$ через $r$, а $O B$ через $r^{\prime}$. Пусть точка $P$ лежит на произвольном штрихе решетки и имеет координаты $x$ и $y$ (рис. $1, a$ ). Тогда функция оптической длины пути примет вид

$$
F=A P+P B+m \lambda n(y) .
$$



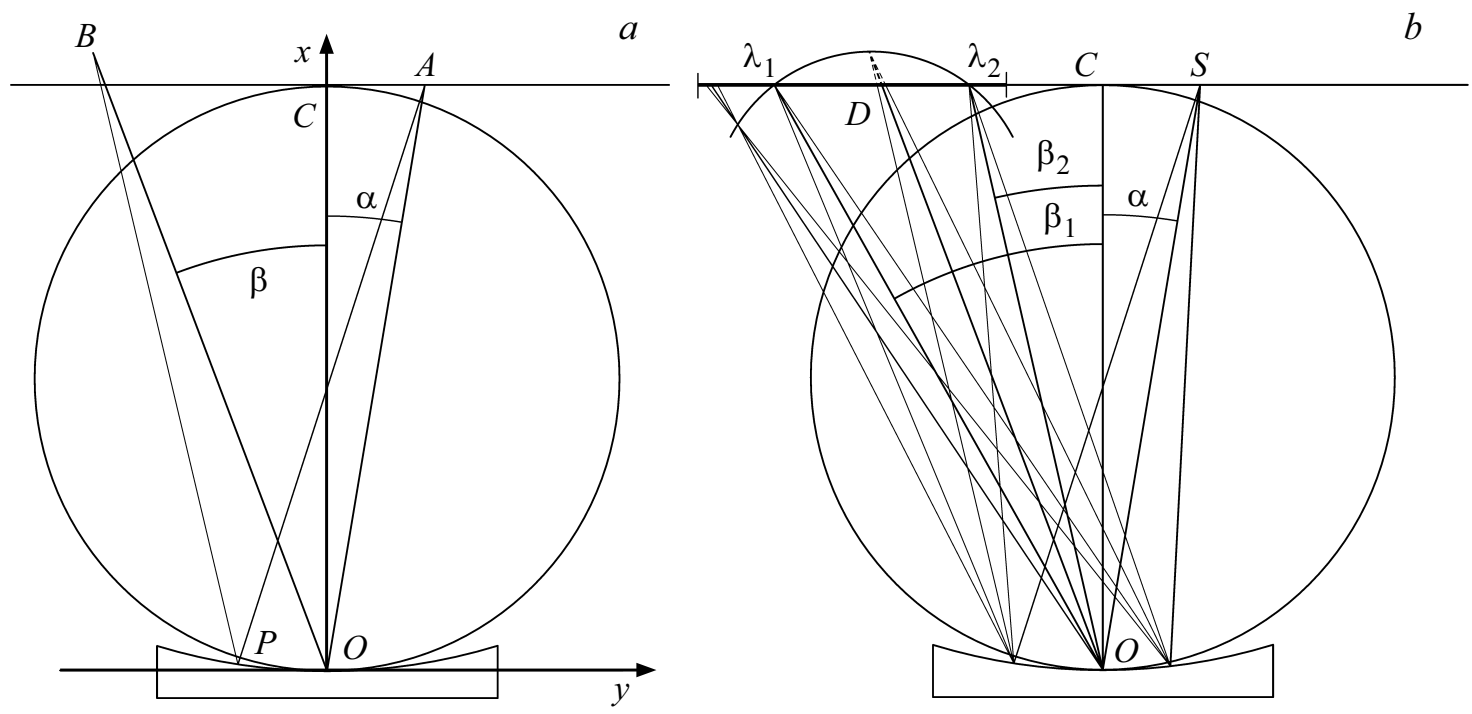

Pис. 1. (a) Система координат в главной плоскости дифракции VLS-решетки. $A-$ точечный источник, $B-$ его спектральное изображение на длине волны $\lambda, P$ - точка, лежащая на одном из штрихов решетки, $C$ - центр кривизны поверхности VLSрешетки, $O$ - центр апертуры VLS-решетки. Круг Роуланда проходит через точки $O$ и $C$, касательная к нему в точке $C-$ ось симметрии решетки. (b) Схема спектрографа нормального падения с компенсацией астигматизма в широком спектральном диапазоне. S - входная щель, D - детектор, $\alpha$ - угол падения центрального луча на VLS-peшетку. $\beta_{1}$ и $\beta_{2}-$ углы дифракции, на которых скомпенсирован астигматизм, $\lambda_{1}$ и $\lambda_{2}-$ соответствующие им длины волн. Все углы правее центральной нормали положительны, левее - отрицательны.

Обозначим угол падения центрального луча на решетку через $\alpha$, а угол дифракции через $\beta$, что дает возможность выразить $A P^{2}$ и $P B^{2}$ :

$$
\begin{aligned}
A P^{2} & =(r \cos \alpha-x)^{2}+(r \sin \alpha-y)^{2} \\
& =r^{2}+x^{2}+y^{2}-2 r(x \cos \alpha+y \sin \alpha), \\
P B^{2} & =\left(r^{\prime} \cos \beta-x\right)^{2}+\left(r^{\prime} \sin \beta-y\right)^{2} \\
& =r^{\prime 2}+x^{2}+y^{2}-2 r^{\prime}(x \cos \beta+y \sin \beta) .
\end{aligned}
$$

Поскольку точка $P$ находится на сферической поверхности решетки радиуса $R$, то

$$
(x-R)^{2}+y^{2}=R^{2} .
$$

Выражая из (5) $x$ через $y$, подставляя его в (3), (4), а затем извлекая корень, пользуясь разложением в ряд из работы [15], после применения принципа Ферма $(d F / d y=0)$ и приведения подобных по степеням $y$ слагаемых, получим

$$
\begin{gathered}
\sin \alpha+\sin \beta=m p_{0} \lambda, \\
\frac{\cos ^{2} \alpha}{r}+\frac{\cos ^{2} \beta}{r^{\prime}}-\frac{\cos \alpha+\cos \beta}{R}+m p_{1} \lambda=0 .
\end{gathered}
$$

Соотношение (6) - уравнение дифракционной решетки, а выражение (7) - условие фокусировки в горизонтальном направлении. При фиксированном положении источника (параметрах $\alpha$ и $r$ ) (6) описывает угол дифракции центрального луча $\beta$, а выражение (7) задает для этого угла дифракции расстояние от центра решетки до горизонтального фокуса $r^{\prime}$. Система из этих уравнений определяет спектральную фокальную кривую $r^{\prime}(\beta)$, форма которой зависит от коэффициента $p_{1}$ VLS-решетки, положения источника и радиуса кривизны решетки. Все фокальные кривые (вне зависимости от значения $p_{1}$ ) будут проходить через точку, соответствующую фокусу нулевого порядка. При $p_{1}=0$ одно из решений уравнения (7) описывает ситуацию, когда и источник, и его изображение находятся на круге Роуланда:

$$
r=R \cos \alpha, \quad r^{\prime}=R \cos \beta .
$$

Важно, что касательная к кругу Роуланда (8) в точке $C$ (рис. 1) является осью симметрии решетки, и любая плоскость, содержащая эту ось, будет эквивалентна главной плоскости. Поэтому каждый луч, вышедший из точки оси симметрии, после дифракции обязательно на нее вернется. Отсюда следует, что точечный источник, расположенный на касательной, будет отображен на плоскости, содержащей эту касательную, в виде тонкого отрезка, толщина которого определяется только дифракционной расходимостью и зависит от вертикальной апертуры решетки и длины волны.

Ось симметрии является важным частным случаем вертикальной фокальной кривой и описывается уравнением

$$
r=\frac{R}{\cos \alpha}, \quad r_{v}^{\prime}=\frac{R}{\cos \beta},
$$

где $r_{v}^{\prime}$ - расстояние от центра решетки до вертикального фокуса. Дифракционное качество вертикального изображения при расположении точечного источника 
на оси симметрии позволяет реализовать высокое пространственное разрешение при компенсации астигматизма. Поэтому в рассматриваемых далее оптических схемах на основе сферической VLS-решетки входная щель и плоскость детектора будут располагаться на этой прямой.

В случае решетки с криволинейными штрихами входная щель располагается на круге Роланда, поэтому спектральная фокальная кривая $r_{h}^{\prime}(\beta)$ описывается уравнением (8). Кривизна штриха приводит к изменению формы вертикальной фокальной кривой, уравнение которой имеет вид

$$
\frac{1}{r}+\frac{1}{r_{v}^{\prime}}-\frac{\cos \alpha+\cos \beta}{R}+\frac{\sin \alpha+\sin \beta}{R_{g}}=0,
$$

где $R_{g}$ - радиус кривизны штриха [12].

\section{Совмещение спектральной и вертикальной фокальных кривых в двух точках}

Пусть входная щель располагается на оси симметрии сферической VLS-решетки. Тогда в силу (9) вертикальное изображение центральной точки входной щели также будет лежать на этой оси. Поэтому для получения стигматизма нужно модифицировать спектральную фокальную кривую так, чтобы она пересекла эту прямую. Сделать это можно, подобрав коэффициент $p_{1}$ зависимости (1) частоты штрихов VLS-решетки.

Пусть стигматизм выполняется для угла дифракции $\beta$ на длине волны $\lambda$. Тогда в этой точке уравнение (7) с учетом (9) примет вид

$$
\frac{\cos ^{3} \alpha+\cos ^{3} \beta-\cos \alpha-\cos \beta}{R}+m p_{1} \lambda=0 .
$$

Удовлетворить условию стигматизма (11) оказывается возможным одновременно на двух углах дифракции (рис. 1,b). Пусть точки пересечения фокальных кривых соответствуют углам дифракции $\beta_{1}$ и $\beta_{2}$, причем $\beta_{2}>\beta_{1}$. При этом оптическая схема прибора примет вид, изображенный на рис. $1, b$. Входная щель и детектор располагаются на оси симметрии решетки - касательной к кругу Роуланда. Излучение с длинами волн $\lambda_{1}$ и $\lambda_{2}$ дифрагирует под углами $\beta_{1}$ и $\beta_{2}$ и фокусируется в точках пересечения спектральной фокальной кривой и оси симметрии, давая стигматическое изображение входной щели на плоскости детектора. Излучение с другими длинами волн фокусируется на поверхность детектора лишь в вертикальном направлении. Для центра спектрального диапазона горизонтальный фокус расположен чуть дальше оси симметрии, а для краев - чуть ближе, давая при этом на детекторе линии конечной ширины. Эта ширина определяет спектральное разрешение прибора и зависит от величины астигматической разности и рабочей ширины решетки. Угол падения $\alpha$, длины волн стигматических точек $\lambda_{1}$ и $\lambda_{2}$ и коэффициент $p_{1}$ можно найти из системы уравнений (11) и (6), записанных для каждого из углов дифракции $\beta_{1}$ и $\beta_{2}$ :

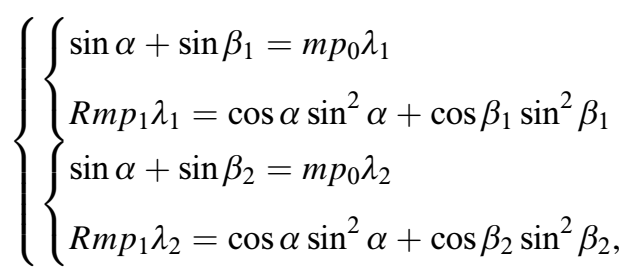

Исключая $m \lambda_{1,2}$ из системы (12), ее можно свести к уравнению

$$
\begin{aligned}
\frac{R p_{1}}{p_{0}} & =\frac{\cos \alpha \sin ^{2} \alpha+\cos \beta_{1} \sin ^{2} \beta_{1}}{\sin \alpha+\sin \beta_{1}} \\
& =\frac{\cos \alpha \sin ^{2} \alpha+\cos \beta_{2} \sin ^{2} \beta_{2}}{\sin \alpha+\sin \beta_{2}} .
\end{aligned}
$$

Приближенное решение этого уравнения, применимое при нормальном падении, позволяет в явном виде получить зависимость угла падения $\alpha$ от $\beta_{1}$ и $\beta_{2}$ :

$$
\sin \alpha \approx \frac{\sqrt{b^{2}-2 c \sqrt{4 a^{2}-2 b^{2}+4 a c+2 b \sqrt{b^{2}-4 a c}}}-b}{\sqrt{4 a^{2}-2 b^{2}+4 a c+2 b \sqrt{b^{2}-4 a c}}},
$$

где

$$
\begin{gathered}
a=\sin \beta_{2}-\sin \beta_{1}, \\
b=\sin ^{2} \beta_{1} \cos \beta_{1}-\sin ^{2} \beta_{2} \cos \beta_{2}, \\
c=\sin \beta_{1} \sin \beta_{2}\left(\sin \beta_{1} \cos \beta_{1}-\sin \beta_{2} \cos \beta_{2}\right) .
\end{gathered}
$$

Из (14) следует, что угол падения $\alpha$, удовлетворяющий уравнению (13), всегда больше, чем $\beta_{1}$. Коэффициент $p_{1}$ может быть найден подстановкой (14) в уравнение (13).

Из (13)-(15) следует, что при фиксированных значениях радиуса кривизны решетки $R$ и частоты штрихов в центре ее апертуры $p_{0}$ расположение входной щели и требуемый градиент частоты штрихов $p_{1}$ однозначно определяется углами дифракции, соответствующими точкам пересечения спектральной и вертикальной фокальных кривых. Поскольку при этом также однозначно задается и форма спектральной фокальной кривой, то все свойства оптической схемы в главной плоскости (в том числе спектральный диапазон и максимальная геометрическая расфокусировка, накладывающая ограничение на достижимое спектральное разрешение прибора) будут зависеть только от выбора этих углов дифракции $\beta_{1}$ и $\beta_{2}$.

Аналогичным образом можно найти условия совмещения спектральной и вертикальной фокальной кривой на двух углах дифракции для сферической периодической решетки с криволинейными штрихами. В этом случае следует составить систему из уравнений (6), (8) и (10), записанных для углов дифракции $\beta_{1}$ и $\beta_{2}$, и потребовать 
на них выполнения равенства $r_{h}^{\prime}\left(\beta_{1,2}\right)=r_{v}^{\prime}\left(\beta_{1,2}\right)$. Отсюда получается уравнение

$$
\begin{gathered}
\frac{\left(\sin \alpha+\sin \beta_{1}\right) \cos \beta_{1}}{-\sin ^{2} \alpha \cos \beta_{1}-\sin ^{2} \beta_{1} \cos \alpha}=\frac{R_{g}}{R} \\
=\frac{\left(\sin \alpha+\sin \beta_{2}\right) \cos \beta_{2}}{-\sin ^{2} \alpha \cos \beta_{2}-\sin ^{2} \beta_{2} \cos \alpha},
\end{gathered}
$$

которое имеет приближенное решение

$$
\sin \alpha \approx \frac{\sqrt{b^{2}-\frac{8 a^{2} c}{\sqrt{4 a^{2}-2 b^{2}+4 a c+2 b \sqrt{b^{2}-4 a c}}}-b}}{\frac{4 a^{2}}{\sqrt{4 a^{2}-2 b^{2}+4 a c+2 b \sqrt{b^{2}-4 a c}}}},
$$

где

$$
\begin{gathered}
a=\cos \beta_{1} \cos \beta_{2}\left(\sin \beta_{2}-\sin \beta_{1}\right), \\
b=\sin ^{2} \beta_{1} \cos \beta_{2}-\sin ^{2} \beta_{2} \cos \beta_{1}, \\
c=\sin \beta_{1} \sin \beta_{2}\left(\sin \beta_{1} \cos \beta_{2}-\sin \beta_{2} \cos \beta_{1}\right) .
\end{gathered}
$$

(18) Подстановкой угла падения $\alpha$ из (17) в уравнение (16) находится необходимое для выполнения стигматизма значение радиуса кривизны штриха. Вид выражений (16)-(18) свидетельствует о том, что, как и в случае сферической VLS-решетки с прямолинейными штрихами, все свойства схемы в главной плоскости зависят только от выбранных углов стигматических точек.

\section{Основные свойства стигматической схемы нормального падения}

Наиболее важными характеристиками схемы нормального падения с компенсацией астигматизма являются максимальная по рабочему диапазону астигматическая разность $\delta_{\text {ast }}$, определяющая максимальную геометрическую расфокусировку, относительная ширина спектрального диапазона $\Delta \lambda_{s} / \lambda_{s}$ min и расстояние между входной щелью и ближайшим к ней краем детектора $\Delta_{\mathrm{SD}}$. Приведем для них выражения:

$$
\begin{aligned}
& \delta_{\text {ast }}=r^{\prime}-r_{v}^{\prime} \\
& =R\left(\frac{\cos ^{2} \beta_{0}}{\cos \alpha-\cos ^{3} \alpha+\cos \beta_{0}-\frac{R p_{1}}{p_{0}}\left(\sin \alpha+\sin \beta_{0}\right)}-\frac{1}{\cos \beta_{0}}\right) \\
& \quad \frac{\Delta \lambda_{s}}{\lambda_{s} \min }=\frac{\max \left(\lambda_{1}, \lambda_{2}\right)-\min \left(\lambda_{1}, \lambda_{2}\right)}{\min \left(\lambda_{1}, \lambda_{2}\right)} \\
& =\frac{\max \left(\sin \alpha+\sin \beta_{1}, \sin \alpha+\sin \beta_{2}\right)}{\min \left(\sin \alpha+\sin \beta_{1}, \sin \alpha+\sin \beta_{2}\right)} \\
& \Delta_{\mathrm{SD}}=R\left(\tan \alpha-\tan \beta_{2}\right)
\end{aligned}
$$

где $\beta_{0}=\operatorname{atan}\left[0.5\left(\tan \beta_{1}+\tan \beta_{2}\right)\right]-$ угол дифракции, для которого расфокусировка максимальна. Заметим, что в выражениях (19) и (21) $R$ выступает в качестве сомножителя и определяет только абсолютный масштаб. Коэффициент $R p_{1} / p_{0}$, согласно уравнению (12), также будет одним и тем же для разных радиусов кривизны VLS-решетки при заданных $\beta_{1}$ и $\beta_{2}$. Поэтому далее астигматическая разность и расстояние $\delta_{\mathrm{SD}}$ будут приведены относительно радиуса кривизны решетки, т.е. в виде $\delta_{\text {ast }} / R$ и $\Delta_{\mathrm{SD}} / R$. Величина $\delta_{\text {ast }} / R$ имеет еще один физический смысл. Его произведение на ширину решетки есть максимальная по рабочему диапазону ширина спектрального изображения центральной точки входной щели на плоскости детектора.

Для анализа свойств схемы спектрографа в зависимости от выбранных углов дифракции, для которых скомпенсирован астигматизм, удобнее перейти к параметрам $\beta_{1}$ и $\Delta \beta=\beta_{2}-\beta_{1}>0$. Рассмотрим в этих обозначениях каждую из характеристик (19)-(21).

Чем больше $\Delta \beta$, тем шире будет рабочий спектральный диапазон и тем больше астигматическая разность (19). При этом ее значение практически не зависит от $\beta_{1}$. Графики зависимости астигматической разности (19) от $\beta_{1}$ при двух сильно разных значениях $\beta_{1}\left(-8.30^{\circ}\right.$ и $\left.0.65^{\circ}\right)$ приведены на рис. $2, a$. Зависимость относительной ширины спектрального диапазона от $\beta_{1}$ и $\Delta \beta$ имеет более сложный вид. Линии постоянного значения этой функции на координатной плоскости с осями $\beta_{1}$ и $\Delta \beta$ напоминают веер прямых, исходящих из точки вблизи начала координат (рис. $2, b$ ).

Расстояние $\Delta_{\mathrm{SD}}$ может служить практическим ограничением ширины спектрального диапазона. Такая ситуация возникает во внутренних порядках дифракции $(m>0)$ при попытке потребовать ширину спектрального диапазона больше октавы. Во внутренних порядках углы дифракции для длин волн рабочего диапазона положительны. Ширина рабочего спектрального диапазона больше октавы означает, что выполняется $\lambda_{2}-\lambda_{1}>\lambda_{1}$. Тогда из уравнения решетки (6) следует $\sin \alpha+\sin \beta_{2}-\sin \alpha-\sin \beta_{1}>\sin \alpha+\sin \beta_{1}$. Но это неравенство не может быть выполнено, если $\alpha>\beta_{2}$. Поэтому $\beta_{1}<\alpha<\beta_{2}$, т. е. входная щель располагается между точками, соответствующими $\lambda_{1}$ и $\lambda_{2}$ на детекторе (т. е. закрывает собой часть рабочего спектрального диапазона). Исходя из практических соображений, расстояние между входной щелью и детектором должно быть не менее $5 \mathrm{~cm}$, поэтому трудности могут возникать и тогда, когда $\alpha>\beta_{2}$. В таком случае преодолеть их можно с помощью увеличения радиуса кривизны решетки $R$ (а вместе с ним и габаритов прибора) при фиксированных углах падения и дифракции. Для увеличения этого расстояния также можно воспользоваться дополнительным плоским зеркалом в скользящем падении, правда, за счет потери в светосиле при отражении.

Более подробный анализ (21) с учетом (14), (15) показывает, что $\Delta_{\mathrm{SD}}$ в большей степени зависит от $\beta_{1}$, чем от $\Delta \beta$. Во внутренних порядках дифракции это расстояние почти не зависит от $\Delta \beta$, в то время как во внешних его зависимость от $\Delta \beta$ немного более 

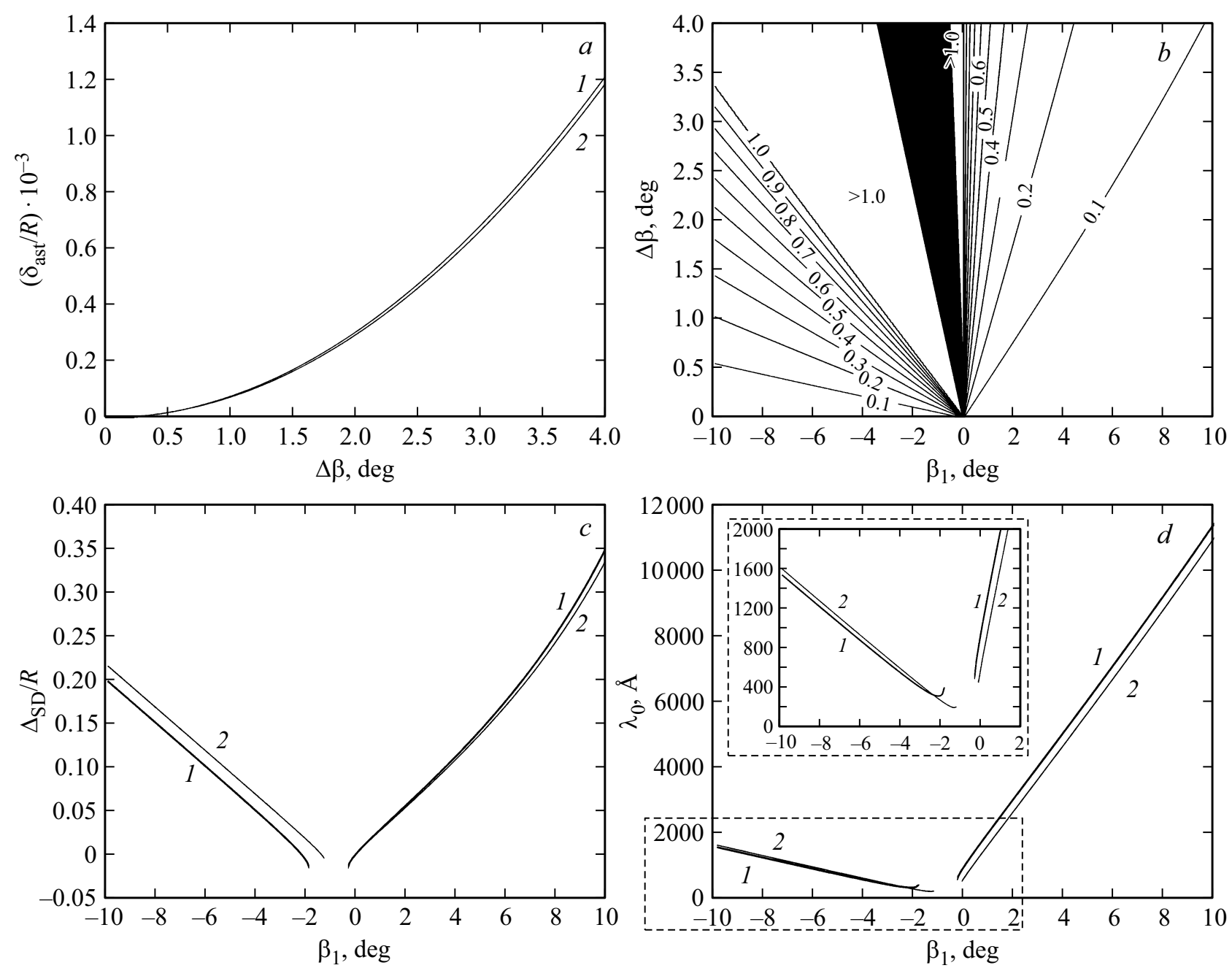

Рис. 2. (a) Зависимость максимальной астигматической разности в рабочем диапазоне от $\Delta \beta$. Кривая $1: \beta_{1}=-8.30^{\circ}$ (внешний порядок дифракции), кривая 2: $\beta_{1}=0.65^{\circ}$ (внутренний порядок дифракции). (b) Относительная ширина спектрального диапазона $\Delta \lambda / \lambda_{\min }(16)$, заключенного между $\lambda_{1}$ и $\lambda_{2}$, в виде карты изолиний в координатах $\beta_{1}$ и $\Delta \beta$. Значение „1.0“ соответствует октаве длин волн. (c) Зависимость расстояния „щель-детектор“ от $\beta_{1}: \Delta \beta=2.10^{\circ}(1), 1.29^{\circ}(2)$. (d) Зависимость длины волны центра спектрального диапазона от $\beta_{1}$ при $p_{0}=600 \mathrm{~mm}^{-1}: \Delta \beta=2.10^{\circ}(1), 1.29^{\circ}(2)$. На врезке - участок, соответствующий вакуумному ультрафиолетовому диапазону, в увеличенном масштабе. Черный треугольник на $(b)$ и разрывы линий на $(c)$ и $(d)$ соответствуют области параметров $\beta_{1}$ и $\Delta \beta$, где уравнение (12) не имеет действительных решений.

существенна. Графики зависимости $\Delta_{\mathrm{SD}}$ от $\beta_{1}$ при двух значениях $\Delta \beta$ приведены на рис. $2, c$.

Значение длины волны $\lambda_{0}$, соответствующей углу дифракции $\beta_{0}$, также зависит от $\beta_{1}$ и $\Delta \beta$. В первом внешнем порядке эта зависимость более пологая, а в первом внутреннем - более крутая. Эта длина волны вместе с $\Delta \lambda_{s} / \lambda_{s}$ min определяет рабочий спектральный диапазон. Графики зависимости $\lambda_{0}$ от $\beta_{1}$ при двух значениях $\Delta \beta$ и $p_{0}=600 \mathrm{~mm}^{-1}$ даны на рис. $2, d$.

Отметим, что левые участки графиков $(b, c, d)$ на рис. 2, занимающие большую часть полуплоскости $\beta_{1}<0$, соответствуют внешним порядкам дифракции, а правые участки - внутренним. При этом угол падения $\alpha$ в каждой точке определяется выражением (14).

Кратное изменение $p_{0}$ приводит к обратно пропорциональному ему кратному сдвигу рабочего спектрального диапазона, но при этом также меняется и дисперсия прибора. Кроме того, коэффициент $p_{0}$, как правило, принимает одно из стандартных значений $(600,1200$, $\left.1800 \mathrm{~mm}^{-1}, \ldots\right)$. Все это делает затруднительным использование $p_{0}$ в качестве степени свободы при подстройке схемы прибора под выбранный диапазон длин волн. Поэтому при расчете оптических схем необходимо выбирать $\beta_{1}$ и $\Delta \beta$ в зависимости от необходимого значения $\lambda_{0}$.

Для схемы на основе периодической решетки с криволинейными штрихами основными характеристиками являются те же самые астигматическая разность $\delta_{\text {ast }} / R$ (которая теперь ограничивает не спектральное, а пространственное разрешение), относительная ширина спектрального диапазона $\Delta \lambda_{s} / \lambda_{s} \min$, расстояние между щелью и детектором $\Delta_{\mathrm{SD}}$ и центральная длина волны $\lambda_{0}$. 
Различие заключается в том, что выражения для астигматической разности и угла дифракции $\beta_{0}$ имеют другой вид:

$$
\begin{gathered}
\beta_{0} \approx 0.5\left(\beta_{1}+\beta_{2}\right), \\
\frac{\delta_{\text {ast }}}{R}=\frac{\cos \alpha}{\cos \alpha \cos \beta_{0}-\sin ^{2} \alpha-\frac{R \cos \alpha(\sin \alpha+\sin \beta)}{R_{g}}}-\cos \beta_{0} .
\end{gathered}
$$

Астигматическая разность (23) при этом имеет в 24 раза большую величину, чем в случае VLS-решетки, но растет более плавно с увеличением $\Delta \beta$. В области внешних порядков дифракции она практически не зависит от $\beta_{1}$, но во внутренних она очень резко нарастает с увеличением $\beta_{1}$ и при этом почти не зависит от $\Delta \beta$. Расстояние $\Delta_{\mathrm{SD}}$ незначительно отличается от такого для схемы с VLS-решеткой, а длина волны $\lambda_{0}$ и относительная ширина спектрального диапазона полностью совпадают с их значениями для VLS-решетки при одних и тех же $\beta_{1}$ и $\Delta \beta$.

\section{Метод расчета оптических схем}

Для расчета приборов использовался следующий алгоритм. Вначале задавались требуемая относительная ширина спектрального диапазона и приблизительная длина волны его центра $\lambda_{0}$, а также апертура решетки. Затем значение астигматической разности $\delta_{\text {ast }} / R$ ограничивалось сверху так, чтобы ширина изображения точечного источника в спектральном направлении при заданной апертуре решетки не превосходила значительно размер ячейки ПЗС-детектора $(\sim 13 \mu \mathrm{m})$. Из этого ограничения находилось требуемое значение $\Delta \beta$. Затем для выбранного значения $\Delta \beta$ подбирались такие значения $\beta_{1}$, при которых длина волны центра рабочего диапазона и относительная его ширина с достаточной точностью соответствовали требуемым. Для этих значений находилось расстояние „щель-детектор“, которое ограничивалось снизу значением $5 \mathrm{~cm}$. Исходя из этого ограничения, выбирался один из оставшихся допустимых углов $\beta_{1}$ и задавался радиус кривизны решетки. Необходимые для этого угол падения $\alpha$ и градиент частоты штрихов $p_{1}$ находились из подстановки параметров $\beta_{1}$ и $\Delta \beta$ в (14) и (13).

Далее производилось построение спектральной фокальной кривой с помощью системы уравнений (6) и (7) и, исходя из ограничения на максимум астигматической разности, определялись границы рабочего спектрального диапазона $\lambda_{\min }$ и $\lambda_{\max }$. При этом ширина диапазона, заключенного между $\lambda_{\min }$ и $\lambda_{\max }$, примерно в полтора раза превосходит ширину диапазона между $\lambda_{1}$ и $\lambda_{2}$. Затем проводилась численная трассировка лучей на опорных длинах волн (края рабочего диапазона $\lambda_{\min }$ и $\lambda_{\max }$, „стигматические точки“ $\lambda_{1}$ и $\lambda_{2}$, а также длина волны центра диапазона $\lambda_{0}$ ) для проверки правильности расчета и анализа спектрального и пространственного разрешений прибора. В качестве точечных монохроматических источников рассматривались точки входной щели, смещенные вдоль ее высоты на 0, 1, 2, 3, 4 и 5 mm относительно центра. Это давало возможность оценить максимально допустимую освещенную высоту щели, а также влияние смещения точечного монохроматического источника от оси симметрии решетки на спектральное и пространственное разрешение. Критерием качества служили ширины изображений точек по основанию в спектральном и пространственном направлениях на плоскости детектора, которые не должны превосходить две ячейки детектора ( 26 $\mu \mathrm{m})$. Аналогичным способом рассчитывались и схемы на основе решетки с криволинейными штрихами.

\section{Результаты расчетов и обсуждение}

Для расчета спектрографов нормального падения были заданы следующие параметры: ширина спектрального диапазона - около октавы, центральная длина волны $\lambda_{0} \sim 1200 \AA, p_{0}=600 \mathrm{~mm}^{-1}$ и апертура решетки (ширина $\times$ высота) $50 \times 50 \mathrm{~mm}$.

Строго удовлетворить этим параметрам в первом внутреннем порядке дифракции оказалось проблематичным. В той области параметров $\beta_{1}$ и $\Delta \beta$, где $\Delta \lambda_{s} / \lambda_{s \text { min }}>0.6$ (этого значения достаточно для получения октавы между $\lambda_{\min }$ и $\left.\lambda_{\max }\right)$, а также $\lambda_{0} \approx 1200 \AA$, расстояние между щелью и детектором составляет менее $0.01 R$. Для соблюдения достаточного расстояния $(5 \mathrm{~cm})$ между ними требовались минимальные габариты прибора более $5 \mathrm{~m}$. Поэтому для реализации полной ширины диапазона был выбран первый внешний порядок дифракции. Этому соответствуют значения $\beta_{1}=-8.3014^{\circ}, \Delta \beta=2.0968^{\circ}$. Необходимый угол падения излучения $\alpha=2.9176^{\circ}$ от нормали. Строгая компенсация астигматизма при этом происходит на длинах волн $\lambda_{1}=1558 \AA$ и $\lambda_{2}=953 \AA$, полный диапазон составляет 820-1690 А. Расстояние „щель-детектор“ $\Delta_{\mathrm{SD}}=5 \mathrm{~cm}$ уже при $R=0.33 \mathrm{~m}$, но из соображений более сильной линейной дисперсии и высокой разрешающей способности радиус кривизны решетки был выбран $R=1 \mathrm{~m}$. Требуемый градиент частоты штрихов $p_{1}=-0.149 \mathrm{~mm}^{-2}$. Основанная на $\delta_{\text {ast }} / R$ оценка максимальной ширины спектрального изображения точечного монохроматического источника в главной плоскости дает результат $17 \mu \mathrm{m}$. Фокальная кривая этой оптической схемы приведена на рис. 3, $a$.

Для расчета прибора, работающего во внутреннем порядке дифракции, относительная ширина рабочего спектрального диапазона была сокращена до $\Delta \lambda_{s} / \lambda_{s \min } \sim 0.35$. При $\lambda_{0} \sim 1200 \AA$ это привело к $\beta_{1}=0.6454^{\circ}, \Delta \beta=1.2195^{\circ}$. Угол падения $\alpha=3.0^{\circ}$. Длины волн „стигматических точек“ $\lambda_{1}=1060 \AA$ и $\lambda_{2}=1435 \AA$. Для получения практически реализуемого расстояния „щель-детектор“, радиус кривизны решетки был выбран $R=5 \mathrm{~m}$. При таком большом радиусе 

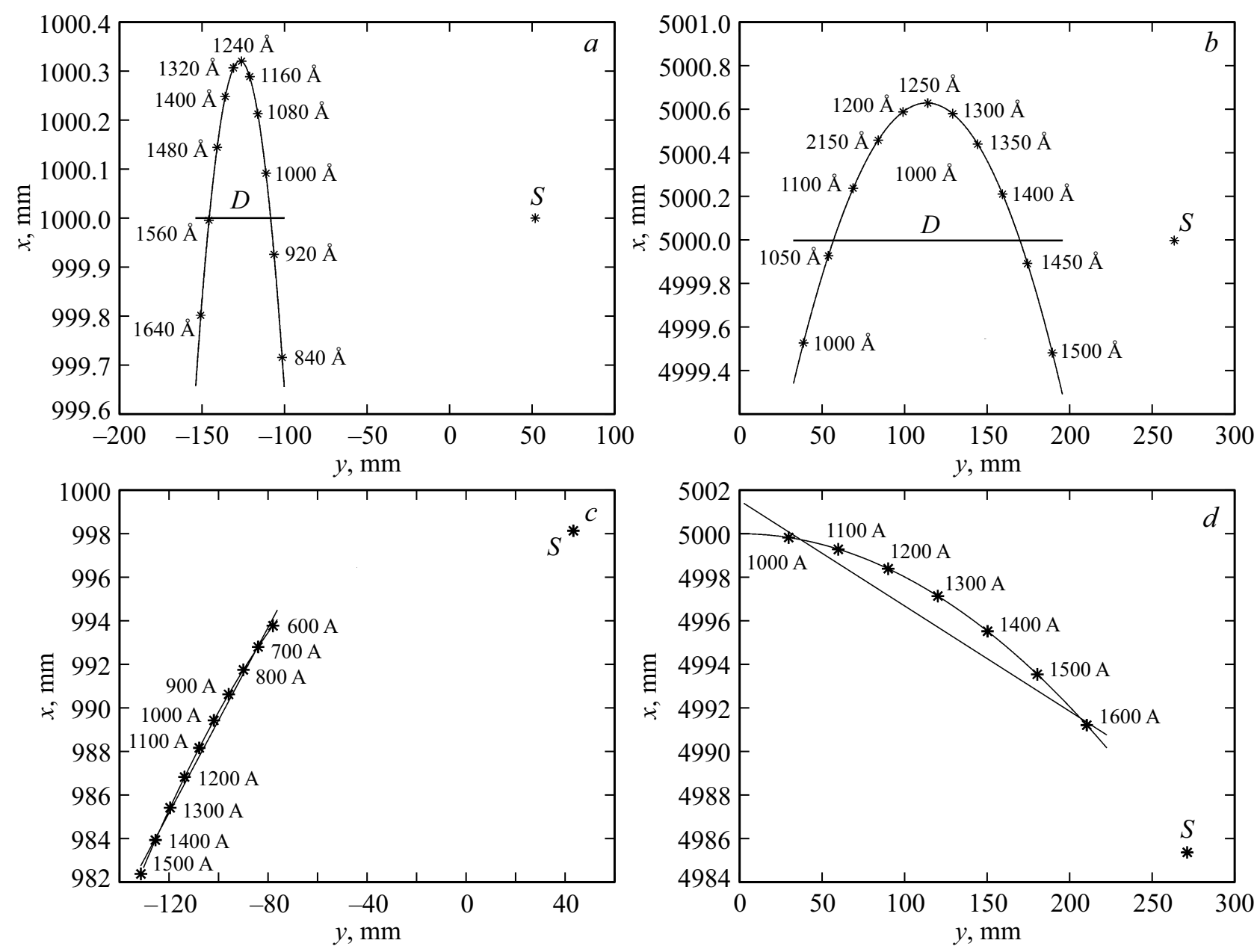

Рис. 3. Взаимное расположение спектральной фокальной кривой (тонкая линия с подписанными длинами волн), плоскости детектора D (отрезок прямой) и входной щели $\mathrm{S}$. Система координат совпадает с рис. 1, $a$ (начало координат расположено в центре апертуры решетки), масштаб по оси $O x$ крупнее, чем по $O y$. (a) Схема, работающая в первом внешнем порядке дифракции, с шириной спектрального диапазона более октавы, $R=1 \mathrm{~m}$. (b) Схема со сверхвысоким разрешением, работающая в первом внутреннем порядке дифракции, $R=5 \mathrm{~m}$. (c) Схема на основе решетки с криволинейными штрихами, работающая в первом внешнем порядке дифракции $(R=1 \mathrm{~m})$. Кривая с нанесенными длинами волн - круг Роуланда, отрезок прямой - вертикальная фокальная кривая, $\mathrm{S}$ - входная щель. (d) Схема на основе решетки с криволинейными штрихами, работающая во внутреннем порядке дифракции $(R=5 \mathrm{~m})$.

кривизны для совмещения спектральной и вертикальной фокальных кривых требуется незначительный градиент частоты штрихов $p_{1}=0.0054 \mathrm{~mm}^{-2}$. Полный рабочий диапазон длин волн при этом составил 980-1520. Астигматическая разность $\delta_{\text {ast }} / R$ оказалась достаточно мала: при ширине решетки $50 \mathrm{~mm}$ ширина спектрального изображения в главной плоскости не превышает $6 \mu \mathrm{m}$ (без вклада дифракционной расходимости), что позволяет получить сверхвысокое спектральное разрешение (рис. 3, b).

Для сравнения был произведен расчет похожих по характеристикам схем на основе периодической решетки с криволинейными штрихами. Во внешнем порядке дифракции были выбраны $\beta_{1}=-7^{\circ}, \Delta \beta=2^{\circ}$, $R=1 \mathrm{~m}$. Этому соответствуют угол падения $\alpha=2.44^{\circ}$, радиус кривизны штрихов $R_{g}=4.7 \mathrm{~m}$ и длины волн стигматических точек $\lambda_{1}=718 \AA$ и $\lambda_{2}=1370 \AA$. Полная рабочая область спектра при этом составила 570-1500 А. Максимальная астигматическая разность оказалась на уровне $0.3 \mathrm{~mm}$, что при квадратной апертуре решетки со стороной $5 \mathrm{~cm}$ должно приводить к высоте изображения центральной точки входной щели на уровне $15 \mu \mathrm{m}$ на длине волны $\lambda_{0}=1000 \AA$ (рис. 3,c). Во внутреннем порядке дифракции были заданы $\beta_{1}=0.4^{\circ}, \Delta \beta=2^{\circ}, R=5 \mathrm{~m}$. При этом $\alpha=3.1^{\circ}$, $R_{g}=-102.4 \mathrm{~m}, \lambda_{1}=1024 \AA, \lambda_{2}=1587 \AA$, а полная рабочая область спектра составила 910-1640 А. Максимальная астигматическая разность соответствует длине волны $\lambda_{0}=1300 \AA$ и составляет $1.5 \mathrm{~mm}$, что приводит к пространственному размеру центральной точки входной щели на уровне $15 \mu \mathrm{m}$ (рис. $3, d$ ). Спектральные аберрации в обеих схемах пренебрежимо малы по сравнению 
с вкладом дифракционной расходимости, которая для метровых схем дает размер изображения $\sim 20 \lambda$, а для пятиметровых $\sim 100 \lambda$.

Результаты численной трассировки лучей для схем на основе VLS-решетки находятся в согласии с приведенными выше расчетами. В пространственном направлении в главной плоскости аберрации в изображении отсутствуют, откуда следует, что ширина изображения точки в этом направлении будет определяться дифракционной расходимостью. Для метровой схемы во внешнем порядке дифракции она составит $\sim 20 \lambda$, а для пятиметровой во внутреннем порядке $\sim 100 \lambda$. В направлении дисперсии дифракционный предел обычно достигается лишь в окрестности длин волн $\lambda_{1}$ и $\lambda_{2}$, где практически отсутствует геометрическая расфокусировка. Согласно результатам численной трассировки лучей, меридиональная кома незначительна: ее вклад в ширину изображения меньше вклада дифракционной расходимости.

Численная трассировка лучей позволила проанализировать зависимость ширин изображения точечного монохроматического источника от расстояния до центра щели при выносе источника из главной плоскости. Ширина изображений точек входной щели (и в направлении дисперсии, и в пространственном направлении) растет линейно с увеличением расстояния до центра щели. Во внешнем порядке дифракции этот рост происходит довольно быстро: уже при выходе из главной плоскости на $3 \mathrm{~mm}$ ширины изображений возрастают на $\sim 20 \mu \mathrm{m}$ (рис. 4, $a$ и таблица). Во внутреннем порядке этот рост незначителен: в лучевом приближении даже при расстоянии $5 \mathrm{~mm}$ от источника до главной плоскости ширины изображений увеличиваются не более чем на $2 \mu \mathrm{m}$ (рис. $4, b)$.

Для пятиметровой схемы, работающей во внутреннем порядке дифракции, увеличение ширин спектрального изображения при смещении источника от главной плоскости (от оси симметрии) незначительно и никак не сказывается на его спектральном и пространственном разрешении. В данном частном случае также несущественной оказывается и геометрическая расфокусировка: лучевое приближение дает наибольшую ширину изображения $8 \mu \mathrm{m}$, что меньше, чем влияние дифракционной расходимости $(10 \mu \mathrm{m})$. Поэтому пятиметровая схема спектрографа во всем рабочем спектральном диапазоне будет давать изображение дифракционного качества. Ширины изображения по основанию будут составлять $10-15 \mu \mathrm{m}$. Оценка разрешающей способности (при освещении двух ячеек детектора - $26 \mu \mathrm{m}$ ) дает величину $~ 14000$. Пространственное разрешение будет на уровне $26 \mu \mathrm{m}$. Повысить разрешающую способность прибора одновременно с его светосилой можно с помощью увеличения апертуры решетки до размеров, когда совместное влияние расфокусировки и удаления источника от главной плоскости будет сопоставимо с дифракционной расходимостью. Но такую решетку будет сложнее изготовить.
Размеры спектрального изображения (в $\mu \mathrm{m})$ точечного монохроматического источника, полученные с помощью численной трассировки лучей для метровой схемы спектрографа. Учтено влияние дифракционной расходимости

\begin{tabular}{|c|c|c|c|c|c|c|}
\hline \multirow{2}{*}{$\begin{array}{c}\text { Расстояние } \\
\text { от источника } \\
\text { до главной } \\
\text { плоскости, } \\
\text { mm }\end{array}$} & $\lambda, \AA$ & \multirow[t]{2}{*}{820} & \multirow[t]{2}{*}{953} & \multirow[t]{2}{*}{1260} & \multirow[t]{2}{*}{1558} & \multirow[t]{2}{*}{1690} \\
\hline & Направление & & & & & \\
\hline \multirow[t]{2}{*}{0} & Спектральное & 17.2 & 1.9 & 16.0 & 3.1 & 17.0 \\
\hline & Пространственное & 1.6 & 1.9 & 2.5 & 3.1 & 3.4 \\
\hline \multirow[t]{2}{*}{1} & Спектральное & 23.9 & 8.5 & 23.6 & 11.8 & 26.2 \\
\hline & Пространственное & 7.6 & 8.6 & 8.7 & 9.8 & 10.2 \\
\hline \multirow[t]{2}{*}{2} & Спектральное & 29.7 & 16.3 & 31.5 & 21.3 & 35.7 \\
\hline & Пространственное & 15.4 & 15.0 & 17.3 & 19.6 & 20.6 \\
\hline \multirow[t]{2}{*}{3} & Спектральное & 37.3 & 23.6 & 39.7 & 29.7 & 44.2 \\
\hline & Пространственное & 23.0 & 23.0 & 27.2 & 29.5 & 30.9 \\
\hline \multirow[t]{2}{*}{4} & Спектральное & 42.2 & 30.2 & 47.2 & 39.0 & 53.0 \\
\hline & Пространственное & 30.8 & 33.0 & 36.7 & 39.7 & 41.7 \\
\hline \multirow[t]{2}{*}{5} & Спектральное & 48.7 & 36.7 & 54.2 & 47.5 & 63.0 \\
\hline & Пространственное & 39.2 & 41.6 & 45.8 & 50.4 & 52.7 \\
\hline
\end{tabular}

Для метровой схемы, работающей в первом внешнем порядке дифракции, ширины изображений определяются влиянием расфокусировки и сильно возрастают при удалении источника от главной плоскости (таблица). Поэтому для достижения высокого разрешения (спектрального и пространственного) необходимо ограничить освещенную высоту входной щели. Ее оптимальная полувысота, при которой размер изображения в обоих направлениях начинает превышать две ячейки детектоpa $(26 \mu \mathrm{m})$, равна $2.5 \mathrm{~mm}$. Тогда ее пространственное разрешение составит $26 \mu \mathrm{m}$, а спектральная разрешающая способность в центре рабочего диапазона будет на уровне $\sim 2800$. С учетом конечной ширины входной щели $\sim 10 \mu \mathrm{m}$ разрешающая способность будет на уровне $\sim 2500$. Таким образом, эта схема спектрографа обладает высоким разрешением в достаточно широком (более октавы) диапазоне длин волн.

Отметим, что схема c VLS-решеткой допускает лишь незначительную перестройку рабочего спектрального диапазона (за счет изменения угла $\alpha$ ) и в этом смысле является узкоспециализированной. Пятиметровая схема обладает сверхвысоким спектральным и пространственным разрешением, однако ее светосила будет невелика в силу больших габаритов прибора и малых приемных углов. Метровая схема, работающая во внешнем порядке дифракции, способна обеспечить меньшее (но при этом высокое) спектральное и пространственное разрешение в более широком спектральном диапазоне и при большей светосиле.

Схемы на основе решетки с криволинейными штрихами обладают приблизительно такими же характеристиками. Согласно результатам численной трассировки лучей, на длине волны $\lambda_{0}=1000 \AA$ в схеме, работаю- 

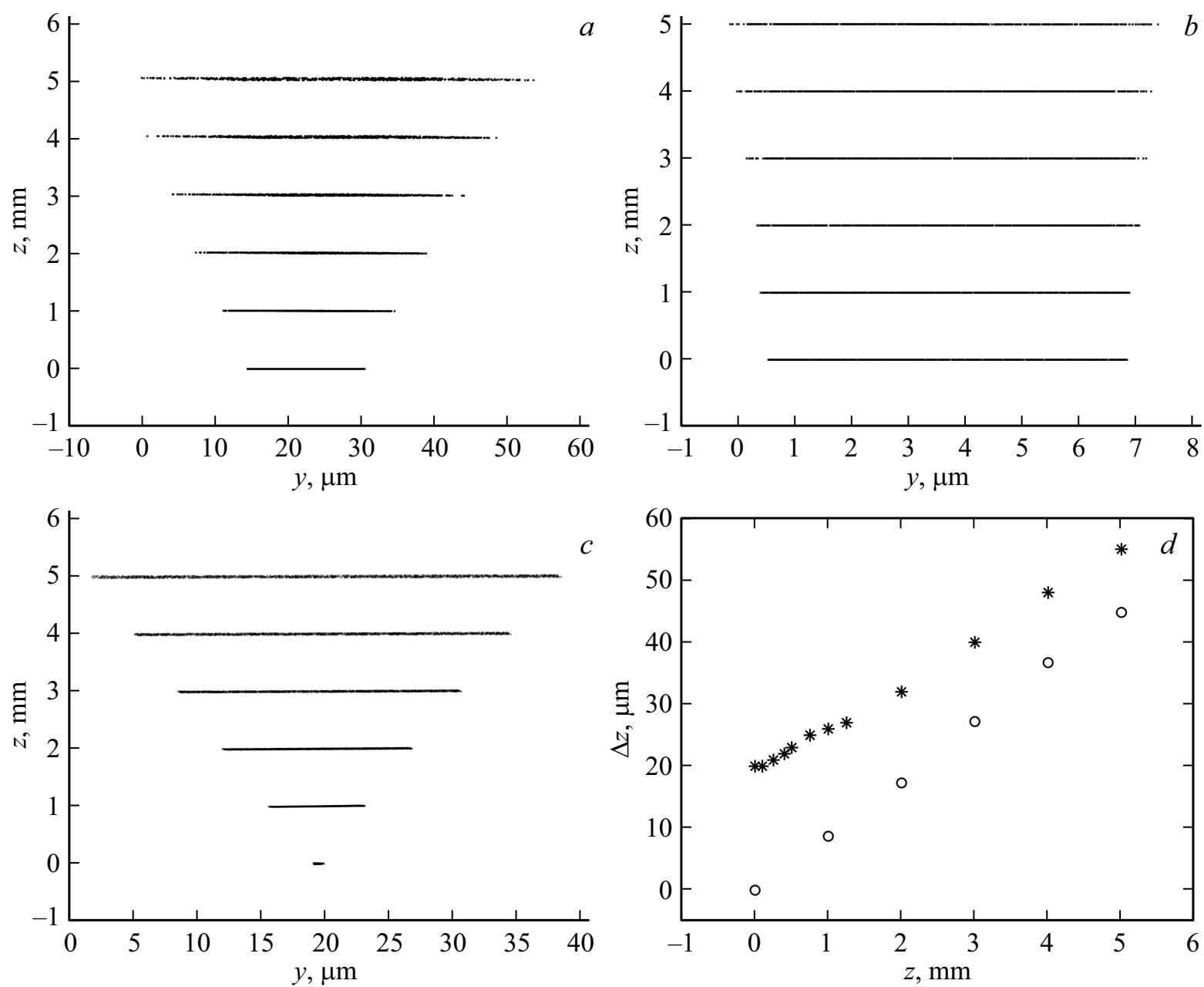

Рис. 4. Спектральные и пространственные аберрации схем с компенсацией астигматизма на длине волны наибольшей астигматической разности $\lambda_{0}$. $(a),(b),(c)$ Изображения шести точек входной щели, отстоящих друг от друга на $1 \mathrm{~mm}$. Показаны спектральные аберрации. $z=0$ соответствует центру входной щели (при этом источник лежит в главной плоскости) для следующих схем: $(a)$ метровая схема с VLS-решеткой, работающая в первом внешнем порядке дифракции, $\lambda_{0}=1260 \AA$; (b) пятиметровая схема c VLS-решеткой, работающая в первом внутреннем порядке дифракции, $\lambda_{0}=1250 \AA$; $(c)$ схема на основе решетки с криволинейными штрихами, работающая в первом внешнем порядке дифракции, $\lambda_{0}=1000 \AA$. $(d)$ Сравнение пространственных аберраций схем, работающих во внешнем порядке дифракции. $\Delta z$ - ширина вертикального изображения точки входной щели, смещенная на $z$ от главной плоскости. Кружками показаны аберрации для схемы с VLS-pешеткой $\left(\lambda_{0}=1260 \AA\right)$, звездочками - для схемы на основе решетки с криволинейными штрихами $\left(\lambda_{0}=1000 \AA\right)$.

щей во внешнем порядке дифракции $(R=1 \mathrm{~m})$, ширина изображения центральной точки входной щели в вертикальном направлении составляет $20 \mu \mathrm{m}$, что означает пространственное разрешение прибора на уровне двух ячеек детектора - $26 \mu \mathrm{m}$. На длинах волн компенсации астигматизма пространственная аберрация мала по сравнению с дифракционным пределом. Разрешающая способность схемы с учетом размера ячейки детектора составляет $\sim 2500$. Схема, работающая во внутреннем порядке дифракции $(R=5 \mathrm{~m})$, дает на длине волны $\lambda_{0}=1300 \AA$. пространственную аберрацию на уровне $16 \mu \mathrm{m}$, что больше дифракционного предела. Спектральная разрешающая способность этой схемы с учетом размера ячейки детектора $\sim 15000$. При смещении то- чечного источника от главной плоскости вдоль входной щели аналогично схемам с VLS-решеткой происходит линейное увеличение пространственной и спектральной аберраций (рис. 4,c и 4,d), что приводит к ограничению поля зрения метрового прибора на уровне $2.5 \mathrm{~mm}$ (в пересчете на угол $\sim 0.143^{\circ}$ ). Для прибора во внутреннем порядке дифракции это ограничение незначительно - линейное поле зрения $\sim 50 \mathrm{~mm}$ (угловое $\sim 0.573^{\circ}$ ). Такими же полями зрения обладают и аналогичные схемы на основе VLS-решетки. Получается, что при использовании ПЗС-детектора между схемами на основе VLS-решетки и схемами на основе решетки с криволинейными штрихами разницы нет. Однако если требуется получение лучшего пространственного 
разрешения, то схема c VLS-решеткой дает по всему спектру в достаточно малом поле зрения вертикальное изображение дифракционного качества, что заведомо не будет выполнено для произвольной длины волны в схеме на основе решетки с криволинейными штрихами. Если же требуется лучшее спектральное разрешение, то более предпочтительной будет схема на основе решетки с криволинейными штрихами. В силу того, что детектирование спектра производится при этом на круге Роуланда, при малых полях зрения получаются изображения дифракционного качества, а при максимально больших полях зрения примерно в два раза лучше, чем в схеме на основе VLS-решетки. В отличие от VLS-решеток решетки с криволинейными штрихами более универсальны и могут применяться не только в подобранной схеме с компенсацией астигматизма, но и в произвольной классической схеме на круге Роуланда.

Отметим также еще одну особенность схем спектрометров на основе решеток с криволинейными штрихами. Существует возможность поместить детектор не на круге Роуланда, а на вертикальной фокальной кривой, которая имеет очень большой радиус кривизны. В этом случае схема может давать вертикальное изображение дифракционного качества на произвольной длине волны (в пределах малого поля зрения), однако при этом она будет обладать несколько меньшим спектральным разрешением, чем схема на основе VLS-решетки.

Достижение стигматизма в области более коротких длин волн, в частности в диапазоне $\lambda<300 \AA$, возможно при использовании VLS-решеток или решеток с криволинейными штрихами, на которые нанесено многослойное рентгеновское зеркало. Так, с VLS-решеткой с $p_{0}=1200 \mathrm{~mm}^{-1}$ и $R=2 \mathrm{~m}$ при угле падения $\alpha=0.898^{\circ}$ удается компенсировать астигматизм в диапазоне длин волн 115-270 без потери спектрального разрешения (максимальная астигматическая разность в указанном диапазоне составляет $85 \mu \mathrm{m}$, что при любом возможном размере решетки даст расфокусировку не более нескольких микрон в направлении дисперсии). Работы по созданию отражательных дифракционных решеток с многослойным покрытием для коротковолнового диапазона ведутся в различных лабораториях уже в течение ряда лет [16-19].

\section{Заключение}

Показано, что использование сферической VLS-peшетки позволяет строить спектрографы нормального падения с компенсацией астигматизма в широком (порядка октавы) интервале длин волн при сохранении высокого спектрального разрешения во всем рабочем спектральном диапазоне. При этом вертикальное изображение центральной точки входной щели будет обладать дифракционным качеством.
Рассчитаны два варианта схемы спектрографа для ВУФ диапазона. Первый вариант использует внутренний порядок дифракции $m= \pm 1$, что позволяет достичь стигматизм в широком диапазоне (менее октавы, 980-1520 ). Для обеспечения расстояния $5 \mathrm{~cm}$ между входной щелью и детектором приходится выбрать VLSрешетку большого радиуса кривизны $R=5 \mathrm{~m}$, что означает большие габариты прибора и невысокую светосилу. Такая схема будет обладать сверхвысокой $\left(>10^{4}\right)$ разрешающей способностью.

Второй вариант использует внешний порядок дифракции $m=-1$. Такая схема компактна (габариты около $1 \mathrm{~m})$ и стигматична в очень широком спектральном диапазоне 820-1690 (более октавы длин волн), но размеры освещенной высоты щели будут сильно влиять на ее спектральное и пространственное разрешение. Такие схемы обладают большей светосилой, чем схемы, работающие во внутренних порядках (при одинаковой апертуре VLS-решетки). При оптимальной освещенной высоте входной щели схема обеспечивает высокую спектральную разрешающую способность $\sim 2500$.

Результаты сравнения этих схем с аналогичными по характеристикам схемами на основе сферической решетки с криволинейными штрихами показали, что существенной разницы между ними нет. Схемы на основе VLS-решетки могут давать в малых полях зрения пространственное разрешение дифракционного качества, в то время как схемы на основе решетки с криволинейными штрихами позволяют получать спектральное разрешение дифракционного качества при таком же ограничении поля зрения. Возможность разместить детектор на вертикальной фокальной кривой (а не на круге Роуланда) практически полностью снимает разницу между этими схемами. Поэтому вопрос в выборе той или иной схемы в значительной степени сводится к вопросу о том, что проще технически реализовать с высокой точностью: вариацию частоты штрихов на уровне нескольких процентов или решетку с эквидистантными штрихами, радиус кривизны которых в 5-20 раз превышает радиус кривизны поверхности решетки.

При использовании VLS-решеток или решеток с криволинейными штрихами с многослойным покрытием подобные схемы нормального падения могут быть использованы в более коротковолновых спектральных областях $\lambda<300 \AA$. В частности, рассчитана схема стигматического спектрографа с VLS-решеткой на область 115-270 А, в которой стигматизм достигается без потери спектрального разрешения. Ширина спектрального диапазона, сам спектральный диапазон, положение входной щели и отношение максимальной астигматической разности к радиусу кривизны решетки определяются углами дифракции, на которых производится компенсация астигматизма.

Исследование выполнено за счет гранта Российского научного фонда (проект № 14-12-00506). 


\section{Список литературы}

[1] Pirozhkov A.S., Kando M., Esirkepov T.Zh., Gallegos P., Ahmed H., Ragozin E.N., Faenov A.Ya., Pikuz T.A., Kawachi T., Sagisaka A., Koga J.K., Coury M., Green J., Foster P., Brenner C., Dromey B., Symes D.R., Mori M., Kawase K., Kameshima T., Fukuda Y., Chen L., Daito I., Ogura K., Hayashi Y., Kotaki H., Kiriyama H., Okada H., Nishimori N., Imazono T., Kondo K., Kimura T., Tajima T., Daido H., Rajeev P., McKenna P., Borghesi M., D. Neely, Kato Y., Bulanov S.V. // Phys. Rev. Lett. 2012. V. 108. P. 135004.

[2] Kando M., Pirozhkov A.S., Kawase K., Esirkepov T.Zh., Fukuda Y., Kiriyama H., Okada H., Daito I., Kameshima T., Hayashi Y., Kotaki H., Mori M., Koga J.K., Daido H., Faenov A.Ya., Pikuz T., Ma J., Chen L.-M., Ragozin E.N., Kawachi T., Kato Y., Tajima T., Bulanov S.V. // Phys. Rev. Lett. 2009. V. 103. P. 235003.

[3] Samson J.A.R. Techniques of Vacuum Ultraviolet Spectroscopy. NY: Wiley, 1967. 216 p.

[4] Зайдель А.Н., Шрейдер Е.Я. Вакуумная спектроскопия и ее применение. М.: Наука, 1976. 431 с.

[5] Rowland H.A. // Phil. Mag. Ser. 1882. V. 5. № 13 (84). P. 469.

[6] Sirks J.L. // Astronomy a. Astrophys. 1894. V. 13. P. 763.

[7] Cornu M.A. // Comptes Rendus Acad. Sci. 1893. V. 117. P. 1032.

[8] Герасимов Ф.М., Яковлев Э.А., Пейсахсон И.В., Кочелев Б.В. // Опт. и спектр. 1970. Т. 28. В. 4. С. 790.

[9] Harada T., Kita T. // Appl. Opt. 1980. V. 19. N 23. P. 3987.

[10] Kita T., Harada T., Nakano N., Kuroda H. // Appl. Opt. 1983. V. 22. N 4. P. 512.

[11] Harada T., Sakuma H., Takahashi K., Watanabe T., Hara H., Kita T. // Appl. Opt. 1998. V. 37. N 28. P. 6803.

[12] Пейсахсон И.В. Оптика спектральных приборов. Изд. 2-е. Л.: Машиностроение, 1975. 313 с.

[13] Герасимов Ф.М., Яковлев Э.А., Кошелев Б.В. // Опт. и спектр. 1979. Т. 46. С. 1177.

[14] Стариев Г.П., Дубровин А.Н., Савушкин А.В., Травина В.Г., Файнберг Л.М. Усовершенствование фотоэлектрических установок для спектрального анализа. Всесоюзн. конф. „Приборы и методы спектроскопии“, тез. докл., Новосибирск, 1979. С. 38.

[15] Namioka T.J. // J. Opt. Soc. Am. 1959. V. 49. N 5. P. 446.

[16] Rife J.C., Hunter W.R., Barbee T.W., Cruddace R.G. // Appl. Opt. 1989. V. 28. N 15. P. 2984.

[17] Kowalski M.P., Seely J.F., Hunter W.R., Rife J.C., Barbee T.W., Holland G.E., Boyer C.N., Brown C.M., Cruddace R.G. // Appl. Opt. 1993. V. 32. P. 2422.

[18] Hunter W.R. // Experim. Meth. in Phys. Sci. 1998. V. 31. P. 379.

[19] Voronov D.L., Goray L.I., Warwick T., Yashchuk V.V., Padmore H.A. // Opt. Expr. 2015. V. 23. N 4. P. 4771. 